\title{
Remise en état des formes de radoub 2 et 3 de Pontaniou a l'arsenal de Brest
}

\author{
Roland Boutin \\ SID, Direction des travaux maritimes, \\ Le Château, BP 16, 29240 Brest \\ roland.boutin@wanadoo.fr
}

Nos ports, qu'ils soient civils ou militaires, rassemblent des installations complexes, parfois anciennes, qu'il convient de pérenniser et de moderniser afin d'assurer en toute sécurité les services aux navires qui y font escale. Les formes 2 et 3 de l'arsenal de Brest (cf. figure 1) font l'objet d'importants travaux de rénovation qui s'achèveront à l'été 2007. Le chantier vise à lutter contre le vieillissement des ouvrages et à adapter les installations aux nouveaux besoins de la marine du fait de la modernisation de sa flotte.

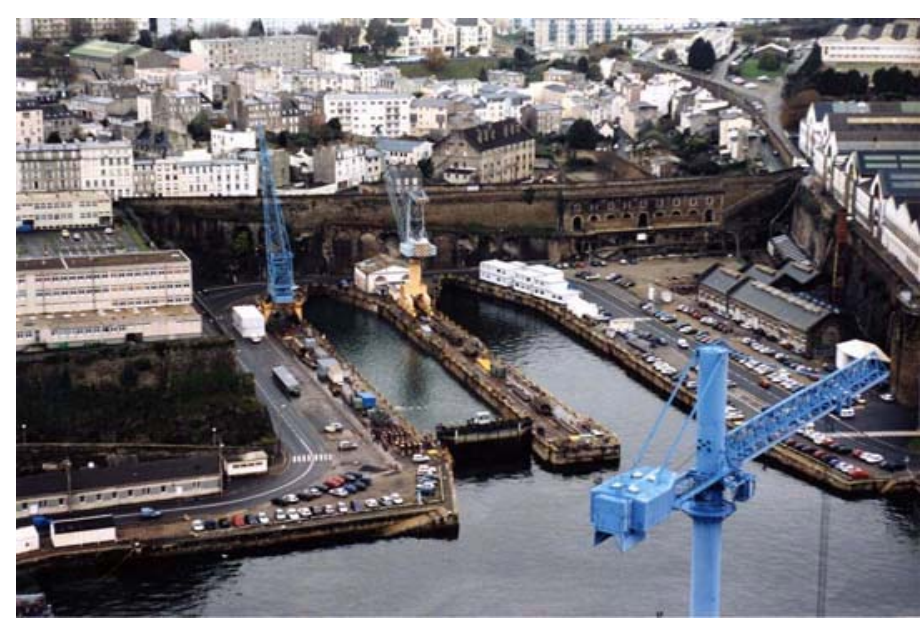

Figure 1 : Vues des formes 2 et 3 de l'arsenal de Brest.

Les formes de raboub $n^{\circ} 2$ et 3 de l'arsenal de Brest sont des constructions du $\mathrm{XVIII}^{\mathrm{e}}$ siècle souvent remaniées. Malgré différents travaux de confortement depuis 1960, l'exploitation des bassins était compromise du fait notamment de la dégradation des maçonneries. Leur remise en état a été décidée en 2000.

Après une phase d'études approfondies, les travaux ont été définis. Ils consistent en la reconstruction des entrées des deux bassins et du môle central sur une longueur de 95 mètres. Le radier du bassin 3 est également démoli jusqu'au rocher pour être approfondi et aplani. Sa nouvelle géométrie est adaptée à l'accueil des nouvelles frégates de la marine française. Pour des raisons architecturales et de rappel historique, les crêtes des bajoyers reconstruits et certaines parties d'ouvrage sont habillées de pierres de tailles provenant des ouvrages anciens. (cf. figure 2). 


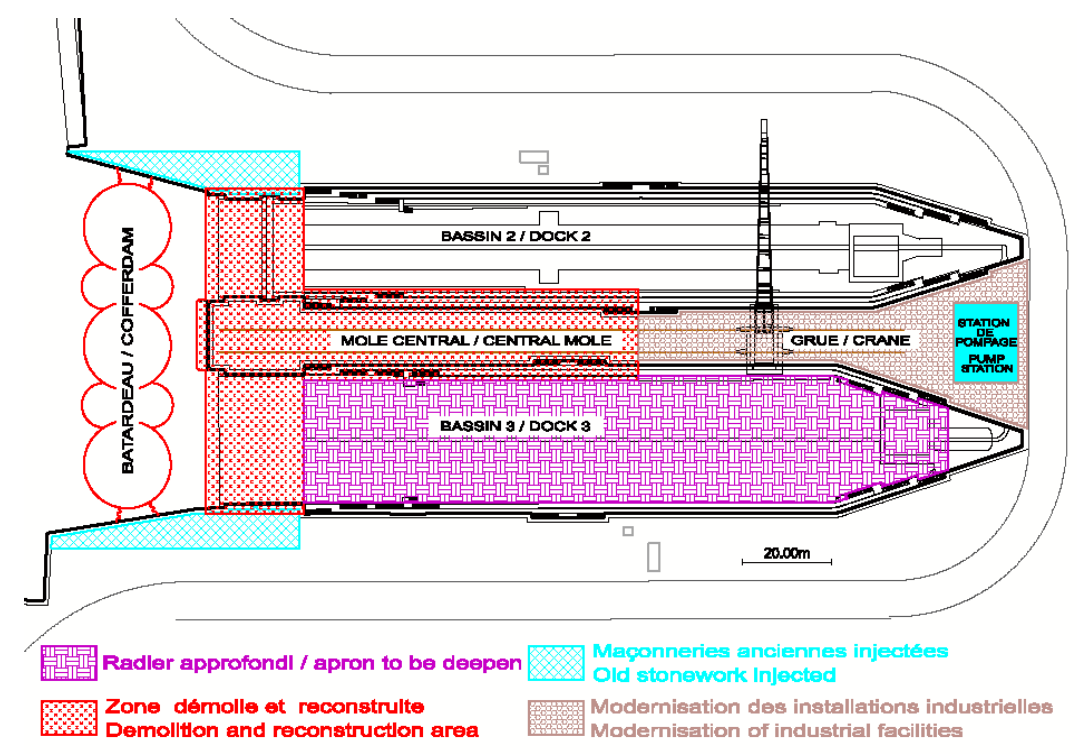

Figure 2 : Travaux de remise en état.

Pour réaliser les travaux de génie civil à "sec", il a été nécessaire de construire un batardeau en avant des ouvrages à reconstruire. Trois gabions circulaires de 19,5 mètres de diamètre reliés par des alvéoles secondaires sont ainsi (cf. figures $3,4,5)$ en palplanches métalliques.

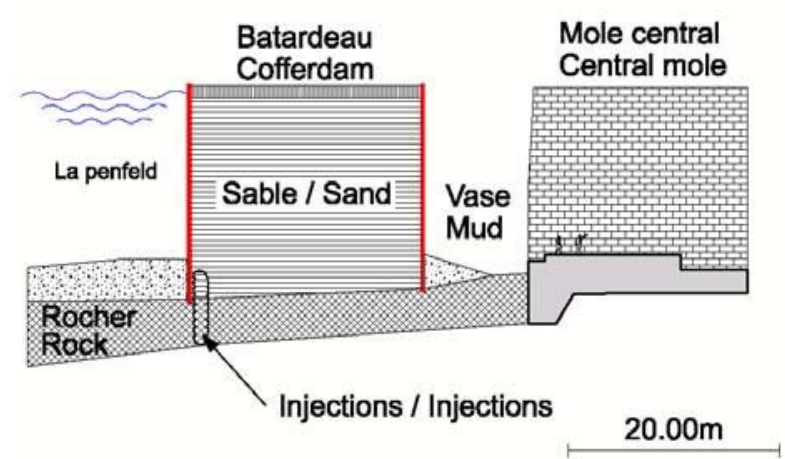

Figure 3 : Coupe de principe du batardeau.
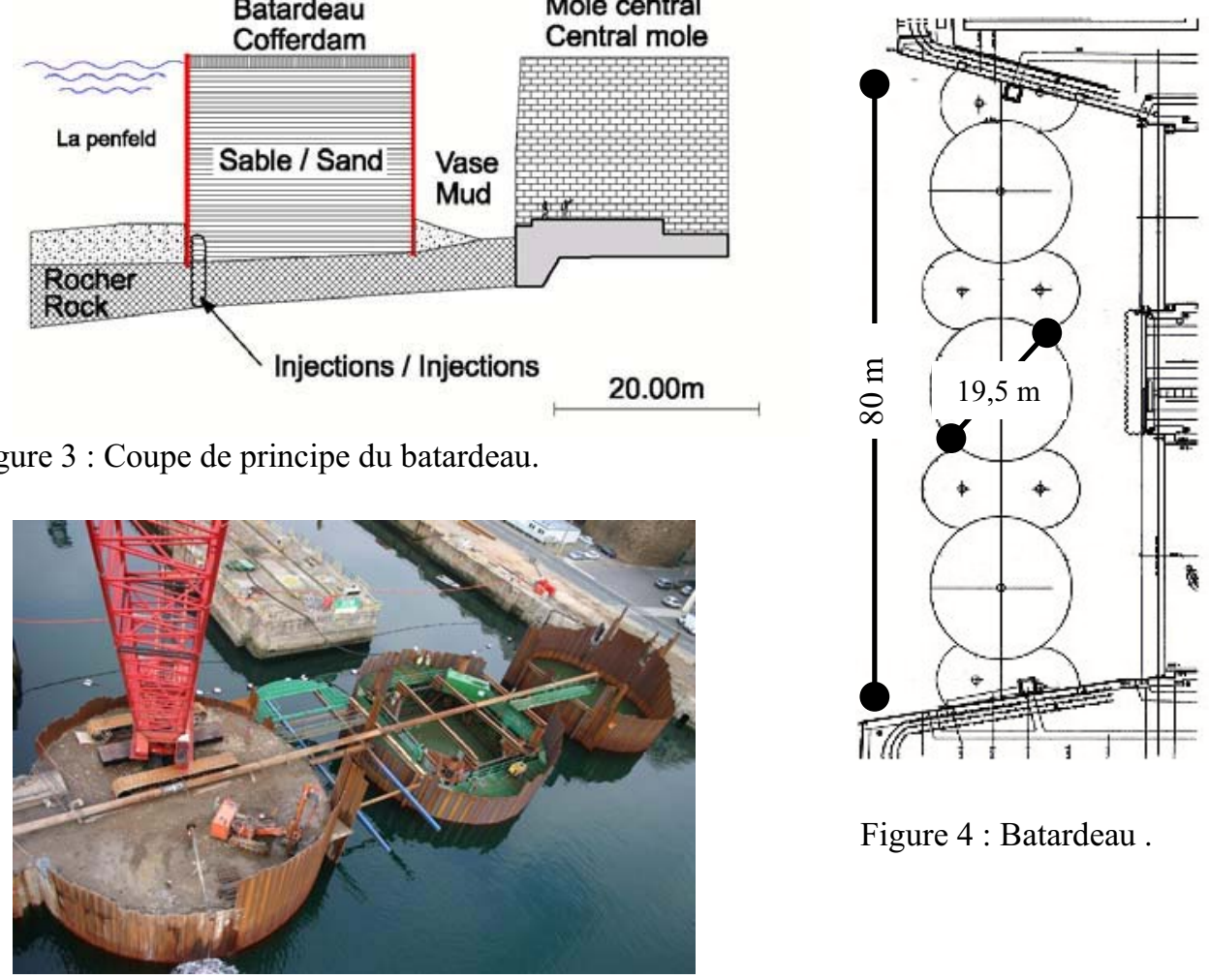

Figure 4 : Batardeau .

Figure 5 : Construction du batardeau. 
La couche de vase en place $\left(2000 \mathrm{~m}^{3}\right)$ au fond des cellules constitue une zone de glissement potentiel peu propice à la stabilité du batardeau. Elle est donc draguée à l'aide de moyens mécaniques ou hydraulique. Trop contaminés pour être clappés en mer, les sédiments sont acheminés vers une unité de traitement (cf. figure 6 et 7), puis évacués par camion dans des sites de stockage à terre agréés. Les cellules sont ensuite remplies de sable $\left(18000 \mathrm{~m}^{3}\right)$.

Figure 6 : Aire de délitage et de scarification.
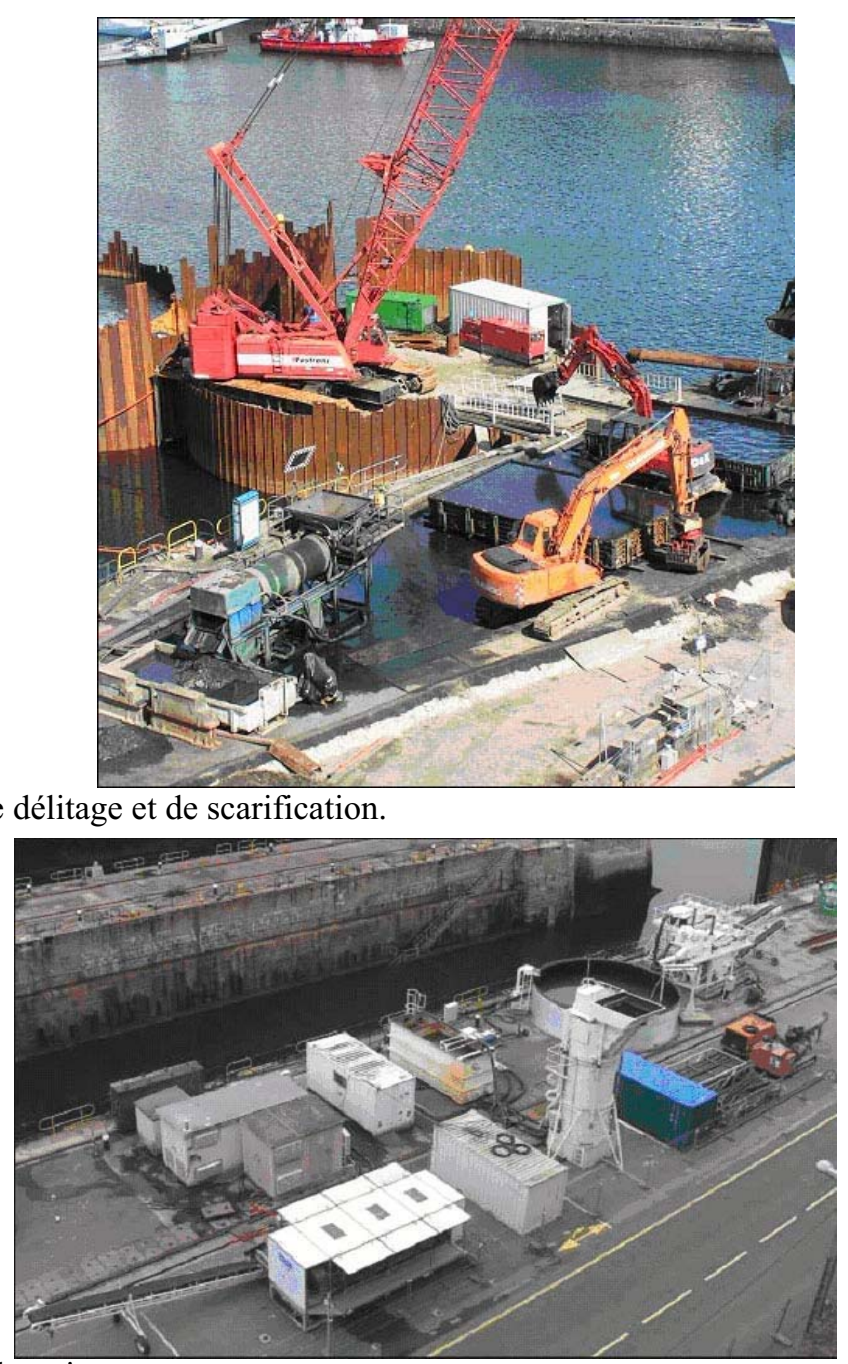

Figure 7 : Unité de traitement.

Le batardeau ainsi réalisé forme une retenue d'eau de plus de 18 mètres de hauteur et assure la sécurité de la suite du chantier. Pour pouvoir détecter tout dysfonctionnement, le batardeau a été instrumenté. Différents capteurs, dont des piézomètres, associés à un système de surveillance piloté par un calculateur, veillent en permanence sur le dispositif.

Un peu moins de neuf mois de travaux auront été nécessaires pour réaliser le batardeau et mettre à sec le chantier

Une fois ce premier chantier achevé, la phase de démolition est engagée. C'est en fait un grand chantier de terrassement (cf. figure 8) qui représente $28000 \mathrm{~m}^{3} \mathrm{de}$ maçonnerie et de déroctage. 
Figure 8 : Démolition.

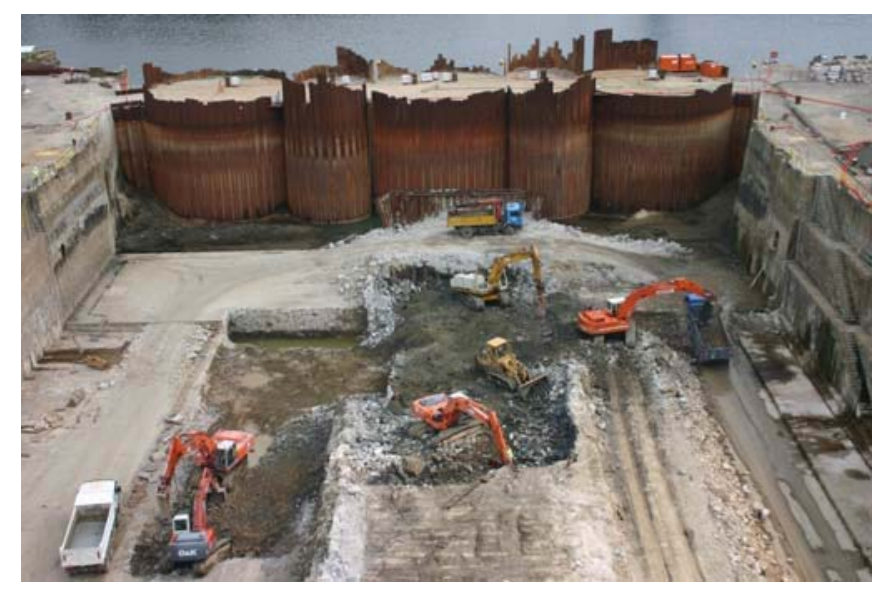

Les travaux de démolition terminés, la construction des nouveaux ouvrages peut s'engager. L'ouvrage reconstruit dans la zone des portes est en forme de U (cf. figure 9) radier et bajoyer. Afin de maîtriser les circulations d'eau à l'entrée des bassins, une bêche formant barrière hydraulique est construite. Une continuité de l'écran hydraulique avec le rocher sous jacent est constituée grâce à 197 forages d'injection percés à travers la bêche et descendus de 4 mètres dans le rocher. 16000 litres de bentonite ciment sont ainsi injectés et viennent colmater la fracturation du rocher.

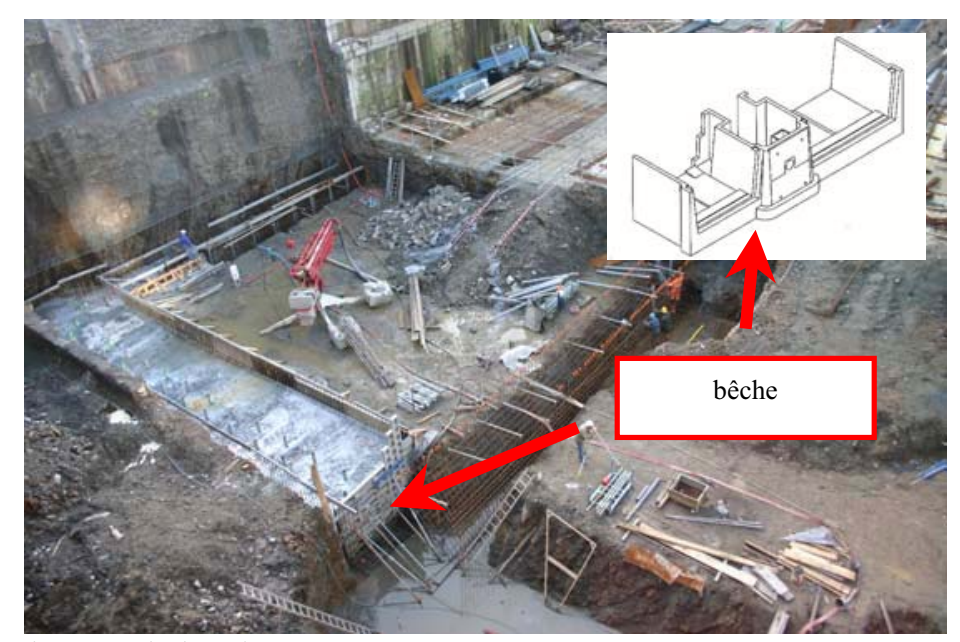

Figure 9 : Zone d'entrée du bassin 2.

En arrière de cet écran, les radiers et l'emprise du môle central sont reconstruits sur une couche drainante en gravier. Un réseau vient collecter les eaux, évitant ainsi toute mise en pression.

Pour la réalisation des ouvrages en béton, aucun joint de dilatation n'est prévu afin de garantir l'étanchéité des bassins. Une formulation à $315 \mathrm{~kg} / \mathrm{m}^{3}$ de ciment avec incorporation de filler calcaire a été préférée.

Afin d'éviter des zones de faiblesse au droit des reprises de bétonnage, les radiers d'entrée ont été réalisés en coulée continue (cf. figure 10). Les $1620 \mathrm{~m}^{3} \mathrm{du}$ 
radier d'entrée du bassin 3 ont été coulés en un peu plus de 20 heures. Cette pièce très massive contient $100 \mathrm{~kg}$ d'acier par mètre cube de béton. Des mesures de températures montrent qu'il faut plus de 15 jours pour dissiper les élévations thermiques.

Figure 10 : Coulage du radier d'entrée du bassin 3.

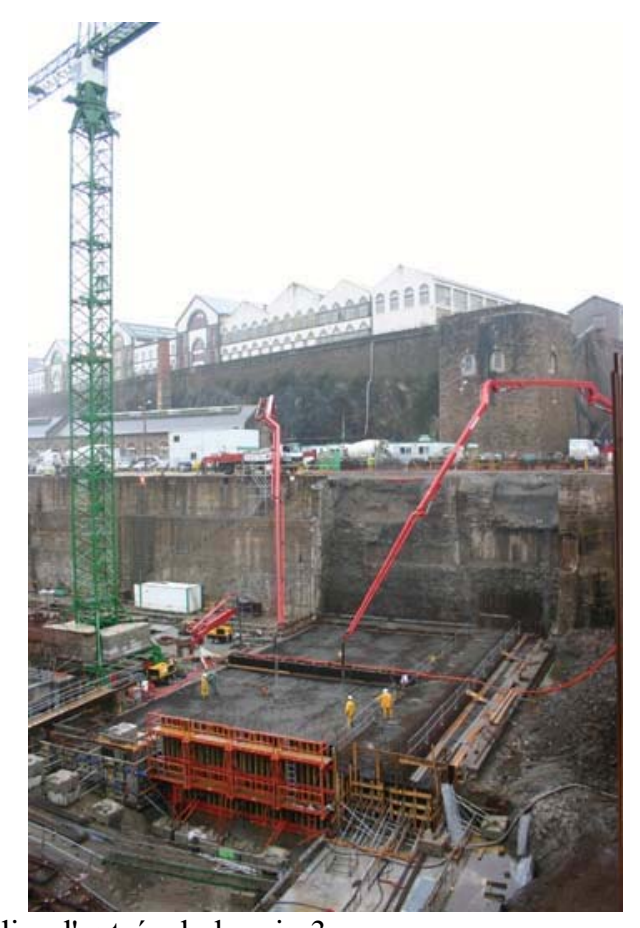

Le radier du bassin 3 est coulé en 12 plots afin de réduire l'effet des phénomènes de retrait. $2700 \mathrm{~m} 3$ de béton sont nécessaires à sa réalisation Des reprises en sous-œuvre sont pratiquées au droit des bajoyers laissés en place. Le môle central est reconstruit également par plots successifs (cf. figures 11 et 12). L'intérieur du môle est quant à lui remblayé avec des matériaux de carrière et des produits issus des démolitions.

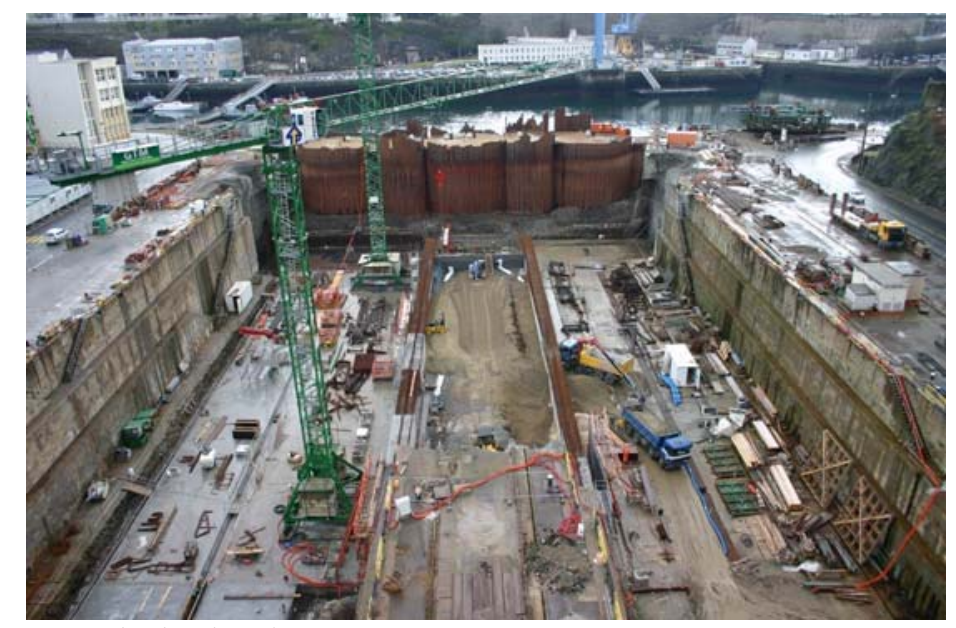

Figure 11 : Vue générale du chantier. 


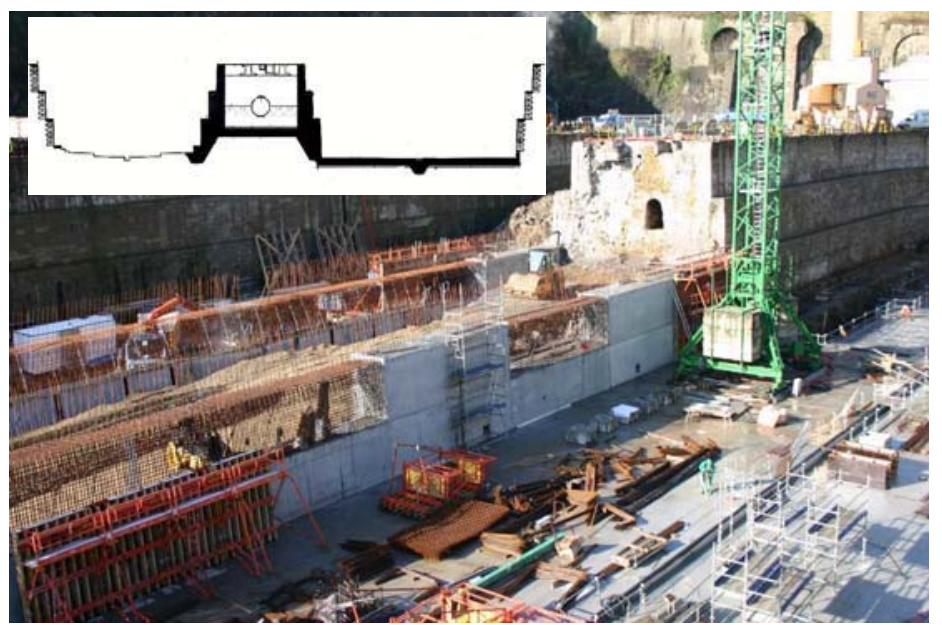

Figure 12 : Reconstruction du môle central.

Il aura fallu environ $16000 \mathrm{~m}^{3}$ de béton, 1800 tonnes d'acier et 13 mois de travaux pour construire les nouveaux bassins. Il restera alors à démonter le batardeau et à remettre les formes en eau.

Les "rénovations" ne touchent pas uniquement le génie civil. La totalité du site "industriel" est concerné. Des équipements de traitement des effluents provenant des travaux de carénage sont installés. Une nouvelle voie de grue est construite sur le môle central. Chaque forme est équipée d'un dispositif de halage, afin de faciliter les manœuvres d'entrée des navires. Le bateau-porte du bassin 2 est caréné et un nouveau bateau-porte pour la forme 3 est en construction. Enfin, la station de pompage devenue obsolète et l'ensemble du réseau d'assèchement et d'épuisement sont entièrement reconfigurés et modernisés. Ces investissements engagés depuis septembre 2004 permettront de répondre à partir de 2007 aux besoins de la marine française.

\section{Conclusions}

Il aura fallu 15 ans pour construire les trois premières formes de Pontaniou. Reconstruites et agrandies au début du XXe siècle, leur dégradation les a peu à peu rendues inexploitables. De nombreux diagnostics et des études approfondies mettant en œuvre des technologies de pointes ont été nécessaires afin de bien appréhender les processus de dégradation de l'ouvrage et prescrire le juste besoin des travaux à réaliser. Un peu plus de deux ans seront nécessaires pour remettre en état et moderniser les formes de Pontaniou.

Nous avons ici une bonne illustration des problématiques actuelles des gestionnaires portuaires. Intervenant sur des ouvrages anciens dégradés, dans des sites fortement industrialisés, ils doivent trouver les bons compromis environnementaux, techniques et économiques pour moderniser et faire vivre les installations. 


\section{Remerciements}

Les travaux présentés sont le fruit de l'engagement de plusieurs générations d'ingénieurs, de techniciens et d'ouvriers. Que ces acteurs, tant publics que privés, soient ici remerciés. C'est leur travail qui est décrit dans cette communication.

\section{Illustrations et crédit photo}

Figure 1: Marine nationale.

Figures 2, 3 et 4 : SID/DTM Brest, François Evano.

Figures 5, 8, 9, 10, 11,12 : SID/DTM Brest, Arnaud Lerouge.

Figures 6, 7 : Société GTM et EXTRACT. 